From theorems 4,8 , and 10 we obtain the following partial answers to that problem: if $X_{0}$ contains only the number $0, X_{1}$ is the set of all non-negative integers, and $X_{2}$ is the set of all non-negative rationals, then $F\left(X_{0}\right)=Q_{2}^{(1)}, F\left(X_{1}\right)=Q_{2}^{(1)}, F\left(X_{2}\right)=\boldsymbol{P}_{3}^{(1)}$.

\title{
References
}

[1] W. Markwald, Zur Eigenschaft primitiv-rekursiver Funktionen, unendlieh viele Werte anzunehmen, this volume, p. 166-167.

[2] A. Mostowski, On definable sets of positive integers, Fund. Math. 34 (1947), p. $81-112$.

Reģu par la Rédaction le 20.9.1954

\section{Contributions to the theory of definable sets and functions}

by

\section{A. Mostowski (Warszawa)}

In this paper we collect some scattered results concerning sets and functions definable in elementary arithmetic. We shall use consistently the terminology and notations of the paper [2], with which, we assume, the reader is acquainted. In particular we denote by $R_{k}$ the set of $k$-ples $\left(x_{1}, x_{2}, \ldots, x_{k}\right)=\mathrm{m}$, where the $x_{j}^{\prime}$ 's are non-negative integers, and by $\boldsymbol{P}_{n}^{(k l)}$ (or $\boldsymbol{Q}_{n}^{(k l)}$ ) the set of functions from $R_{k}$ to $R_{l}$ whose graphs are in $\boldsymbol{P}_{n}^{(k+l)}$ (or in $\boldsymbol{Q}_{n}^{(k+l)}$ ).

1. We begin by establishing some simple properties of the classes $\boldsymbol{P}_{n}^{(k 1)}$ and $\boldsymbol{Q}_{n}^{(k 1)}$.

THEOREM 1. $\boldsymbol{P}_{n}^{(k 1)} \subset \boldsymbol{Q}_{n}^{(k 1)}$.

Proof. The theorem is evident in case $n=0$. Let us, therefore, assume that $n>0$ and $f \in \boldsymbol{P}_{n}^{(k 1)}$. It follows from the definitions that there exists a set $B \in \boldsymbol{Q}_{n-1}^{(k+2)}$ such that

Hence

$$
\{f(\mathrm{~m})=m\} \equiv \sum_{x}\{(\mathrm{~m}, m, x) \in B\} .
$$

$$
\{f(\mathrm{~m}) \neq m\} \equiv \sum_{p, x}\{[(\mathrm{~m}, p, x) \in B] \cdot(p \neq m)\}
$$

which proves that the graph of $f$ is in $Q_{n}^{(k+1)}$, q. e. d.

THEOREM 2. If $n \geqslant 1$, then $\boldsymbol{P}_{n+1}^{(k 1)}-\boldsymbol{Q}_{n}^{(k 1)} \neq 0 \neq \boldsymbol{Q}_{n}^{(k 1)}-\boldsymbol{P}_{n}^{(k 1)}$.

Proof. It is well known that there are sets $M$ which belong to $\boldsymbol{P}_{n+1}^{(k)} \cdot \boldsymbol{Q}_{n+1}^{(k)}$ without belonging to $\boldsymbol{Q}_{n}^{(k)}$. Let $f$ be the characteristic function of such a set $M$. The graph of $f$ is in $\boldsymbol{P}_{n+1}^{(k+1)}$, since

$$
\{y=f(\mathrm{~m})\} \equiv\{(y=0) \cdot(\mathrm{m} \in M)+(y=1) \cdot(\mathrm{m} \in M)\} .
$$

As $\{\mathrm{m} \in M\} \equiv\{f(\mathrm{~m})=1\}$, the graph of $f$ is not in $\boldsymbol{Q}_{n}^{(k+1)}$. Hence $f \in \boldsymbol{P}_{n+1}^{(k 1)}-\boldsymbol{Q}_{n}^{(k 1)}$. Slightly more intricate is the proof that $\boldsymbol{Q}_{n}^{(k 1)}-\boldsymbol{P}_{n}^{(k 1)} \neq 0$. Let $C \in \boldsymbol{Q}_{n}^{(k)}-\boldsymbol{P}_{n}^{(k)}$ and let $B$ be a set in $\boldsymbol{Q}_{n-1}^{(k+1)}$ such that

$$
\mathrm{m} \xi O \equiv \sum_{x}[(\mathrm{~m}, x) \in B] \text {. }
$$


We select an arbitrary point $\mathfrak{m}_{0}$ outside $C$ and put

$$
\begin{gathered}
h(m)=\mathfrak{m}_{0} \quad \text { if } \quad\left\langle s_{1}^{(k)}(m), s_{2}(m)\right) \notin B, \\
h(m)=s_{1}^{(k)}(m) \quad \text { if } \quad\left\langle s_{1}^{(k)}(m), s_{2}(m)\right) \in B,
\end{gathered}
$$

where $s_{1}^{(k)}$ and $s_{2}$ are primitive recursive functions with the property that the formula $m \rightleftarrows\left(s_{1}^{(k)}(m), s_{2}(m)\right)$ establishes a one-one correspondence between elements of $R_{1}$ and elements of $R_{k+1}$.

The set $R_{k}-C$ coincides with the set of values of the function $h$. The graph of $h$ is in $\boldsymbol{P}_{n}^{(k+1)} \cdot \boldsymbol{Q}_{n}^{(k+1)}$, since

$$
\begin{aligned}
\{\mathrm{m}=h(m)\} \equiv\left(\mathfrak{m}=\mathrm{m}_{0}\right) \cdot\left[\left(s_{1}^{(k)}(m), s_{2}(m)\right) \dot{\epsilon} B\right] & + \\
& +\left[\mathrm{m}=s_{1}^{(k)}(m)\right] \cdot\left[\left(s_{1}^{(k)}(m), s_{2}(m)\right) \in B\right] .
\end{aligned}
$$

Let us put

$$
F=\underset{(m, x)}{E}\left\{(\mathrm{~m} \epsilon G) \cdot(x=0)+(x>0) \cdot[\mathrm{m}=h(x-1)] \cdot \prod_{1 \leqslant z<x}[\mathrm{~m} \neq h(z-1)]\right\} .
$$

We have then $F \in Q_{n}^{(k+1)}$, because the set

$$
\underset{(m, x)}{E}\left\{(x>0) \cdot[\mathrm{m}=h(x-1)] \cdot \prod_{1 \leqslant z<x}[\mathrm{~m} \neq h(z-1)]\right\}
$$

belongs to $\boldsymbol{P}_{n}^{(k+1)} \cdot \boldsymbol{Q}_{n}^{(k+1)}$ (see [3], theorem 3.3). From $\mathrm{m} \epsilon C \equiv(\mathfrak{m}, 0) \in F$ we infer that $F \& \boldsymbol{P}_{n}^{(k+1)}$.

We shall show that $F$ is the graph of a function. If $m \in C$, then $(\mathrm{m}, 0) \in F$, and hence $\sum_{x}(\mathfrak{m}, x) \in F$. If $\mathrm{m} \notin C$, there is an integer $y$ such that $\mathrm{m}=h(y)$. Assuming that $y$ is the smallest integer with this property and putting $x=y+1$, we obtain again $(\mathfrak{m}, x), \epsilon F$. Hence the formula $\sum_{x}(\mathrm{~m}, x) \in F$ is true for every $\mathrm{m}$.

It remains to prove that

$$
\left[\left(\mathrm{m}, x_{1}\right) \in F^{\prime}\right] \cdot\left[\left(\mathrm{m}, x_{2}\right) \in F^{\prime}\right] \rightarrow x_{1}=x_{2} .
$$

If $m \in C$, then $x_{1}=0$ and $x_{2}=0$. If $\mathrm{m} \epsilon C$, then $x_{1}>0, x_{2}>0, \mathrm{~m}=h\left(x_{1}-1\right)$ $=h\left(x_{2}-1\right)$, and $\mathrm{m} \neq h(z)$, for every $z<x_{1}-1$ and every $z<x_{2}-1$. It can be easily seen that either of the assumptions, $x_{1}<x_{2}, x_{2}<x_{1}$, leads to contradictions.

The set $F$ is thus shown to be the graph of a function. Since $F \in \boldsymbol{Q}_{n}^{(k+1)}-\boldsymbol{P}_{n}^{(k+1)}$, this function is in $\boldsymbol{Q}_{n}^{(k 1)}$ but not in $\boldsymbol{P}_{n}^{(k 1)}$, q. e. d.

2. In this section we shall establish some properties of the functions of the class $Q_{1}^{(k 1)}$.

Theorem 3. If $f \in \boldsymbol{Q}_{1}^{(k 1)}$, then the set $\underset{(\mathrm{m}, m)}{E}[m \leqslant f(\mathrm{~m})]$ is recursively enumerable ( $i$ e., belongs to the class $\boldsymbol{P}_{1}^{(k+1)}$ ).
Proof. Let $B$ be a recursive set such that

$$
\{f(\mathrm{~m})=m\} \equiv \prod_{x}\{(\mathrm{~m}, m, x) \in B\} .
$$

We hare then the equivalence

$$
\{m \leqslant f(\mathrm{~m})\} \equiv \prod_{j<m} \sum_{x}[(\mathrm{~m}, j, x) \in B],
$$

which proves the theorem.

Remark. If $f$ is the characteristic function of a set $M \in P_{2}^{(k)} \cdot Q_{2}^{(k)}-P_{1}^{(k)}$, then the set $A=E[m \leqslant f(m)]$ is not recursively enumerable since $\mathfrak{m} \in M \equiv(\mathfrak{m}, 1) \in A$. This shows that theorem 1 is, in general, false for functions $f \in \boldsymbol{P}_{2}^{(k 1)}$.

THEOREM 4. If a function $f \in \boldsymbol{Q}_{1}^{(k 1)}$ is majorized by a recursive function, then $f$ is recursive (i.e. belongs to $\boldsymbol{P}_{0}^{(k 1)}$ ).

Proof. Let $B$ be a recursive set such that

$$
\{m=f(\mathrm{~m})\} \equiv \prod_{x}[(\mathrm{~m}, m, x) \in B], \quad B \in \boldsymbol{P}_{0}^{(k+2)}
$$

and $g$ a recursive function such that $f(\mathfrak{m}) \leqslant g(\mathfrak{m})$. Without loss of generality we may assume $B$ to be primitive recursive. We denote by $h$ the characteristic function of $B$ and put

$$
\begin{aligned}
& h^{\prime}(\mathrm{m}, m, 0)=1-h(\mathrm{~m}, m, 0), \\
& h^{\prime}(\mathrm{m}, m, x+1)=[1-h(\mathrm{~m}, m, x+1)]-\sum_{y=0}^{x} h^{\prime}(\mathrm{m}, m, y) .
\end{aligned}
$$

Thus $h^{\prime}$ is a primitive recursive function which vanishes everywhere except in points $(m, m, x)$, where $x$ is the least integer such that $(\mathrm{m}, m, x) \dot{\epsilon} B$.

If $m \neq f(m)$, then there is an $x$ such that $h^{\prime}(\mathfrak{m}, m, x)=1$; no such $x$ exists if $m=f(\mathrm{~m})$. Hence

and the function

$$
\sum_{m=0}^{g(m)} \sum_{x=0}^{\infty} h^{\prime}(\mathrm{m}, m, x)=g(\mathrm{~m})
$$

$$
\gamma(\mathrm{m})=(\mu y)\left[\sum_{m=0}^{g(m)} \sum_{x=0}^{y} h^{\prime}(\mathrm{m}, m, x)=g(\mathrm{~m})\right]
$$

is (general) recursive.

$$
\text { If } m \neq f(\mathrm{~m}) \text { and } m \leqslant g(\mathrm{~m}) \text {, then } \sum_{x=0}^{\gamma(\mathrm{m})} h^{\prime}(\mathrm{m}, m, x)=1 \text {; if } m=f(\mathfrak{m}) \text {, then }
$$
$\sum_{x=0}^{\gamma(m)} h^{\prime}(m, m, x)=0$. Hence we obtain the formula

$$
f(\mathrm{~m})=(\mu m)_{g(\mathrm{~m})}\left[\sum_{x=0}^{\gamma(\mathfrak{m})} h^{\prime}(\mathrm{m}, m, x)=0\right]
$$


in which $(\mu m)_{a}[\ldots]$ denotes the least $m$, satisfying the inequality $m \leqslant a$ and the condition [...] (or 0 , if no such $m$ exists). This formula proves that $f$ is recursive.

3. Let a set $X \subset R_{k+1}$ be such that $\prod_{m} \sum_{m}(m, m) \in X$. A function $f$ is ealled a selector of $X$ if $\prod(\mathrm{m}, f(\mathrm{~m})) \in X$.

THEOREM 5. Recursively enumerable sets possess recursive selectors. Proof. Let $X \in \boldsymbol{P}_{1}^{(k+1)}$ and let $B$ be a recursive set such that

$$
(\mathfrak{m}, m) \in X \equiv \sum_{x}(\mathfrak{m}, m, x) \in B \text {. }
$$

The function

$$
f(\mathrm{~m})=s_{1}^{(1)}\left\{(\mu z)\left[\left(\mathrm{m}, s_{1}^{(1)}(z), s_{2}(z)\right) \in B\right]\right\}
$$

is the required recursive selector of $X$.

THEOREM 6. $g \in Q_{I}^{(k 1)}$ and $g$ is not recursive, the set $\underset{(\mathrm{m}, m)}{E}[m>g(\mathrm{~m})]$ is in $\boldsymbol{Q}_{\mathbf{1}}^{(k+1)}$ and has no recursive selector.

This theorem follows immediately from the theorems 3 and 4.

4. Kleene [1] has constructed two disjoint recursively enumerable sets $X, Y$, such that there is no recursive set $Z$, satisfying the conditions $X \subset Z, Y Z=0$. If in this proof we change the words "recursively enumerable" into "element of $\boldsymbol{P}_{n}^{(k)}$ " and "recursive" into "element of $\boldsymbol{P}_{n}^{(k)} \cdot \boldsymbol{Q}_{n}^{(k)}$ ", we obtain the proof of

THEOREM 7. For each $n>0$ there are disjoint sets $X, Y \in \boldsymbol{P}_{n}^{(k)}$ such that the formulas $\bar{X} \subset Z, Y Z=0$ are not satisfied by any set $Z \in \boldsymbol{P}_{n}^{(k)} \cdot \boldsymbol{Q}_{n}^{(k)}$.

Theorem 7 is not true for sets of class $Q_{n}^{(k)}$. On the contrary, we shall prove

Theorem 8. If $X, Y \in Q_{n}^{(k)}$ and $X Y=0$, then there is a set $Z \in P_{n}^{(k)} \cdot Q_{n}^{(k)}$ such that $X \subset Z$ and $Y Z=0$.

Since the case $n=0$ is evident, we assume that $n>0$ and denote by $M$ and $N$ two sets in $\boldsymbol{P}_{n-1}^{(k+1)}$ such that

(1) $\quad\{\mathrm{m} \in X\} \equiv \prod_{x}\{(\mathrm{~m}, x) \in M\}, \quad\{\mathrm{m} \in Y\} \equiv \prod_{x}\{(\mathrm{~m}, x) \in N\}$.

It follows from $X Y=0$ that $R_{k}=\left(R_{k}-X\right)+\left(R_{k}-Y\right)$ and hence

$$
\prod_{m} \sum_{x}\{[(\mathrm{~m}, x) \in M]+[(\mathrm{m}, x) \in N]\} .
$$

The graph of the function

$$
f(\mathrm{~m})=(\mu x)\{[(\mathrm{m}, x) \notin M]+[(\mathrm{m}, x) \dot{\epsilon} N]\}
$$

way be represented in the form

$$
\underset{(m, x)}{E}[x=f(\mathrm{~m})]=A-B \quad \text { where } \quad A, B \in \boldsymbol{P}_{n-1}^{(k+1)} .
$$

This follows from the equivalence

$$
x=f(\mathrm{~m}) \equiv \prod_{z<x}[(\mathrm{~m}, z) \in M \cdot N] \cdot\{[(\mathrm{m}, x) \in M]+[(\mathrm{m}, x) \in N]\}
$$

and the observation that the sets,

$$
\sum_{(\mathrm{m}, x)} \prod_{z<x}[(\mathrm{~m}, z) \in M \cdot N] \quad \text { and } \quad \underset{(\mathrm{m}, x)}{E}\{[(\mathrm{~m}, x) \in M]+[(\mathrm{m}, x) \in N]\} \text {, }
$$

belong to the classes $\boldsymbol{P}_{n-1}^{(k+1)}$ and $\boldsymbol{Q}_{n-1}^{(k+1)}$ (cf. [3], theorem 3.3). Let ns put

$$
\begin{gathered}
U=\underset{\mathbf{m}}{E}[(\mathrm{~m}, f(\mathrm{~m})) \dot{\epsilon} M], \quad V=\underset{\mathbf{m}}{E}[(\mathrm{~m}, f(\mathrm{~m})) \dot{\epsilon}], \\
Z=r-C .
\end{gathered}
$$

Formula (2) proves that $U+F=R_{k}$. Using (1), we obtain

$$
\mathrm{m} \in X \rightarrow(\mathrm{m}, f(\mathrm{~m})) \in M \rightarrow \mathrm{m} \dot{\epsilon} U
$$

and hence $X \subset R_{k}-U=(U+V)-U=V-U=Z$. In a similar way we show that $Y \subset U-V$ and hence $Y Z=0$.

It remains to evaluate the class of the set $Z$. From (3) and (4) we obtain

$$
\begin{aligned}
\mathrm{m} \epsilon U & \equiv \sum_{x}\{[x=f(\mathrm{~m})] \cdot[(\mathrm{m}, x) \epsilon M]\} \\
& \equiv \sum_{x}[(\mathrm{~m}, x) \epsilon(A-B)-M] \\
& \equiv \prod_{x}\{[x=f(\mathrm{~m})] \rightarrow[(\mathrm{m}, x) \epsilon M]\} \\
& \equiv \prod_{x}\left[(\mathrm{~m}, x) \epsilon B+\left(R_{k+1}-A\right)+\left(R_{k+1}-M\right)\right] .
\end{aligned}
$$

These equivalences prove that $U \in \boldsymbol{P}_{n}^{(k)} \cdot \boldsymbol{Q}_{n}^{(k)}$. In a similar way we prove that $\nabla \in \boldsymbol{P}_{n}^{(k)} \cdot \boldsymbol{Q}_{n}^{(k)}$. It follows from (5) that $Z \in \boldsymbol{P}_{n}^{(k)} \cdot \boldsymbol{Q}_{n}^{(k)}$. Theorem 8 is thus proved.

\section{References}

[1] S. C. Kleene, A symmetric form of Gôdel's theorem, Indag. Math. 12 (1950), p. 244-246.

[2] A. Mostowski, On definable sets of positive integers, Fund. Math. 34 (1947), p. 81-112.

[3] - On a set of integers not definable by means of one-quantifier predicates, An. de la Soc. Pol. de Math. 21 (1948), p. 114-119.

Regu par la Rédaction le 27.9.1954 